\section{LONG RANGE IOT COMMUNICATION}

ALENA GALAJDOVA, ROBERT RAKAY

Technical University of Kosice, Faculty of Mechanical

Engineering, Kosice, Slovakia

DOI: 10.17973/MMSJ.2021_10_2021038

e-mail to corresponding author: alena.galajdova@tuke.sk

The article deals with the design of wireless automation system. The proposed systems compare wireless data transmission devices. The main components and their parameters, which are necessary for building such system and base steps how to create and test a device are described. The created systems can serve as a suitable basis for remote monitoring and control systems in open space applications such as weather system or small-scale home automation system and can be used as an example in the education of students in fields such as Automation or Mechatronics.

KEYWORDS

Long range, wireless communication, micro system, microcontroller.

\section{INTRODUCTION}

Nowadays more and more systems require or support wireless connection. By creating a seamless connection to a remote location, we can control or monitor various devices and systems. This applies to home and industrial automation too. However, the first wireless systems did not support long distance transmissions or required amplified signal transmission and large antennas. Currently available microcontrollers and communication technologies enable remote communication with small mechanical and technical requirements. This type of solution only concerns the monitoring of home automation systems, such as meteorological stations or other measuring systems based on microcontrollers.

Nowadays, the wireless data transmission has replaced wired connection, and the aim of solution is to make everyday life easier and more comfortable for everyone in society [Galajdova 2009, Vagas 2020, Krenicky 2011]. The wireless connection generally allows remote monitoring and control of systems that assist with tasks such as control of lights or heating, ventilation and air conditioning (HVAC), opening and closing of doors, windows and controlling various electrical devices [Simsik 2016, Ketshabetswe 2019].

Every advanced manufacturer is trying to implement some form of wireless interface however not all are suitable for long distance communication. Asset monitoring, security systems, weather stations are just a few of the applications that use long distances wireless communication. All of them can be created using protocols such as: Zigbee, BLE, ESPNow, LoRA, and other RF-based protocols.

Each of these communication tools allows from small to long range, lower transfer rates and connection limitations. Despite the limitations of the above mentioned protocols, they can be integrated to measure temperature, humidity, movement, gas, vibration and similar factors. The transferred data doesn't contain any private information and in the most cases, they are not time-critical nor mission critical [Kar 2017, Vagas 2020].

For the purpose of experimental testing of the system, there is no exact application that would allow remote monitoring, and the devices are tested only as a laboratory model to compare the range of the tested devices and their protocol. The main goal is to create a smooth long range communication channel between transceiver and receiver [Sarga 2018].

The proposed systems consist of transmitting and receiving devices, which are connected first via the LoRa Module and in the second via ESP's ESPNow protocols (Fig. 1). The input of the system is the temperature and humidity sensor DHT11, while in the second experiment only the direct cyclic message is used as information for transmission between the microcontrollers. The measured value is processed and sent, and the signal strength is also measured.

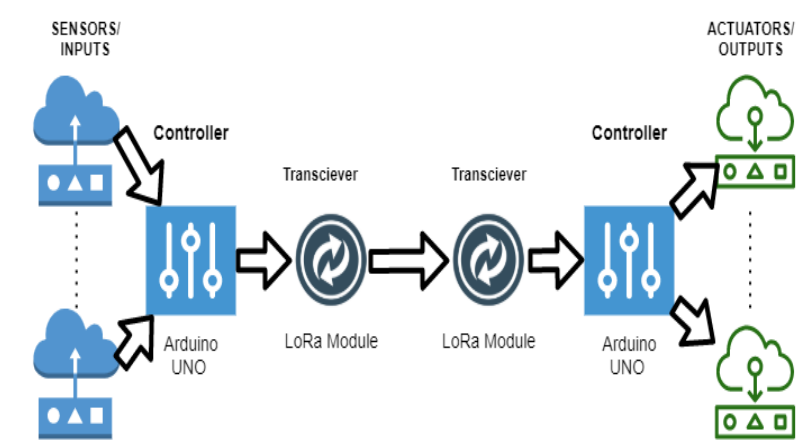

Figure 1. Concept of wireless system with multiple sensors and actuators

\section{LORA MODULE}

LoRa stands for Long range. It is part of LoRaWAN, a longranged energy efficient wireless communication technology, which is defined by the LoRa Alliance.

As the name of the module suggest it is designed for long distance radio communication. It was developed by the AiThinker company. The device uses SX1278 integrated circuit and operates at a frequency of $433 \mathrm{MHz}$. By frequency hopping the range $420-450 \mathrm{MHz}$ is available but up to $525 \mathrm{MHz}$ is supported.

This module works with a power supply of 1.8 to $3.7 \mathrm{~V}$. During the receiving, the power consumption is less than $10.8 \mathrm{~mA}$, while when transmitting, it is less than $120 \mathrm{~mA}$. When the device is in sleep mode, the consumption is $0.2 \mu \mathrm{A}$.

The module uses half-duplex SPI communication, with programmable bit rate, which can reach up to $300 \mathrm{kbps}$ transfer rates. The maximum output is $+22 \mathrm{dBm}$, and the sensitivity is $140 \mathrm{dBm}$.

- $\quad$ ANT Pin is the connector where the spring antenna has to be connected (see Table 1).

- $\quad \mathrm{GND}$ is the ground pin for OV and is usually marked as a reference for identifying the other pins.

- $\quad 3.3 \mathrm{~V}$ is the power pin.

- $\quad$ RESET pin, the module resets itself when powered on. 
- DIO 0-5 universal input output ports on the periphery of the module. The IOs are limited at 3.3 Volts, if additional levels are needed a level conversion circuit must be added.

- $\quad$ SCK (Serial Clock) receives clock pulses provided by the SPI bus Master.

- MOSI (Master Out Slave In) is the SPI input to the module.

- MISO (Master In Slave Out) is the SPI output from the module.

- NSS SPI slave selector pin [LoRa-01 2021].

Table 1. LoRa SX1278 module pins

\begin{tabular}{|l|l|l|}
\hline $\begin{array}{l}\text { Function } \\
\text { Ra-01 }\end{array}$ & Pin & Function Description \\
\hline 1 & ANT & Antenna \\
\hline 2 & GND & GND \\
\hline 3 & $3.3 V$ & $3.3 V$ Power in \\
\hline 4 & RESET & Reset \\
\hline 5 & DIO0 & Digital IO0, software setting \\
\hline 6 & DIO1 & Digital IO1, software setting \\
\hline 7 & DIO2 & Digital IO2, software setting \\
\hline 8 & DIO3 & Digital IO3, software setting \\
\hline 9 & GND & GND \\
\hline 10 & DIO4 & Digital IO4, software setting \\
\hline 11 & DIO5 & Digital IO5, software setting \\
\hline 12 & SCK & SPI Clock input \\
\hline 13 & MISO & SPI data output \\
\hline 14 & MOSI & SPI data input \\
\hline 15 & SPI Selected-IN \\
\hline 16 & GND \\
\hline
\end{tabular}

\section{ESPNOW}

An interesting alternative to creating a local network between devices is the ESPNow protocol. This is a protocol developed by the ESP microcontroller manufacturer that allows multiple devices to be connected without using a Wi-Fi. This protocol is currently under development and there are no further technical specifications.

The protocol is similar to the $2.4 \mathrm{GHz}$ protocol used for lowenergy communication by a wireless mouse. The basis of communication is the pairing of network components, after which the connection is permanent, and no further authentication is required [ESPNow Overview 2021].

As part of this communication, application data is also identified using the OI - identifier of the organization (manufacturer). The CCMP method is used for security, as well as the Primary Master Key and the Local Master Key. MAC addresses are used to address devices. The maximum size of one message is 250 Bytes. The maximum number of elements in one system is 20 unencrypted or 10 encrypted members. Individual participants in the communication can work as the master (controller) of the network, that transmits data, and as a slave (receiving) member that receives and further processes the information.
Because concurrent communication over a Wi-Fi network is not possible when connecting devices, it is an alternative to local data exchange [ESPNow Overview 2021].

\section{ARDUINO UNO R3}

Arduino Uno is a microprocessor board based on ATmega328P. It has 14 digital I/O pins (6 of which can be used as PWM outputs), 6 analog inputs, USB connector, power connector, ICSP header and reset button. It can be easily connected to a computer using a USB cable powered by an AC adapter or a battery [Arduino 2021].

The microcontroller uses an operating voltage $5 \mathrm{~V}$ for inputs and outputs. The maximum current is $20 \mathrm{~mA}$ for $5 \mathrm{~V}$ signals and $50 \mathrm{~mA}$ for $3.3 \mathrm{~V}$ signals. Communication can be performed using UART, I2C or SPI protocols. An external communication module is required for another option.

\section{ESP32}

There are different versions of the ESP development boards available. In our experiment, we will use two ESP32 with an integrated antenna. The following image shows an ESP32.

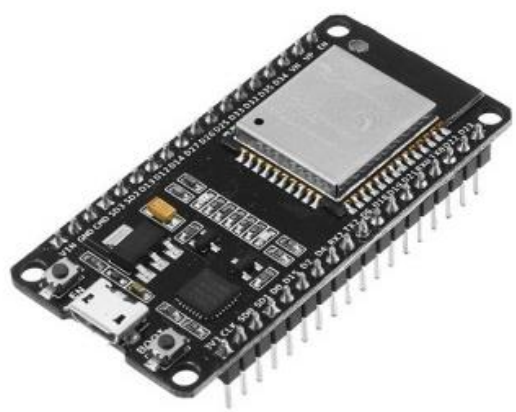

Figure 2. ESP32

The most important technical specifications are listed in the Table 2 below.

Table 2. ESP boards technical specification [ESP32 Datasheet 2021]

\begin{tabular}{|l|l|}
\hline Specs & ESP-32 \\
\hline Power Rating & $459 \mathrm{~mA}$ \\
\hline Core & $4 \times$ Cortex-A53 \\
\hline Architecture & $64-$ bits \\
\hline Clock & $1.4 \mathrm{GHz}$ \\
\hline Wi-Fi & $\mathrm{b} / \mathrm{g} / \mathrm{n} / \mathrm{a}$ c dual band \\
\hline Bluetooth & 4.2 LS BLE \\
\hline RAM & $1 \mathrm{~GB}$ \\
\hline Flash & $1-2$ or 4 GB LPDDR4-3200 RAM \\
\hline GPIO & 40 (28 accessible) \\
\hline DAC & 0 \\
\hline ADC & 0 \\
\hline Interface & SPI-I2C-UART \\
\hline
\end{tabular}




\section{SOFTWARE}

Because we implement Arduino microcontrollers, the Arduino IDE is used as programming environment (Fig. 3). This Java application, that is used to program various microcontrollers such as Arduino, ESP and its clones. The main software includes a text programming code editor, with an integrated code compiler, a tool for code uploading and monitoring functions [Arduino 2021]. The programmed control software includes the following functional parts for library call, different methods of variable declaration, $1 / O$ device and communication configuration and the Main control loop.

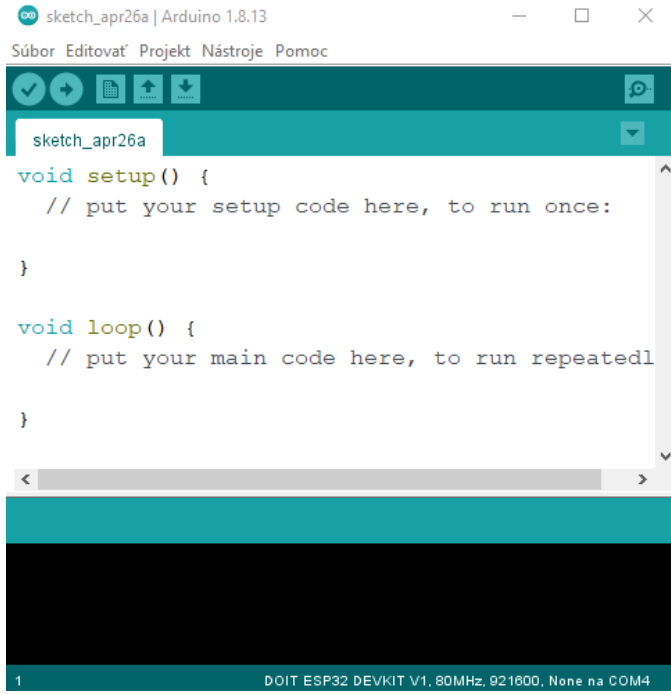

Figure 3. Arduino IDE

First we had to add the Radio-Head library to the development environment. It is a library for embedded microprocessors. It provides a complete object-oriented library for sending and receiving packet messages via various common data radios and other transmissions on a number of built-in microprocessors.

The following figure(Fig.4) is the main part of the software for the module that receives data from the transmitter. After communication between the transmitter and the receiver is established, a "Receive Message" is written to the serial monitor and then the data, is measured on the sensor connected to the transmitter module. If the connection between the transmitter and the receiver is interrupted, the message "Recv Failed" will appear on the serial monitor.

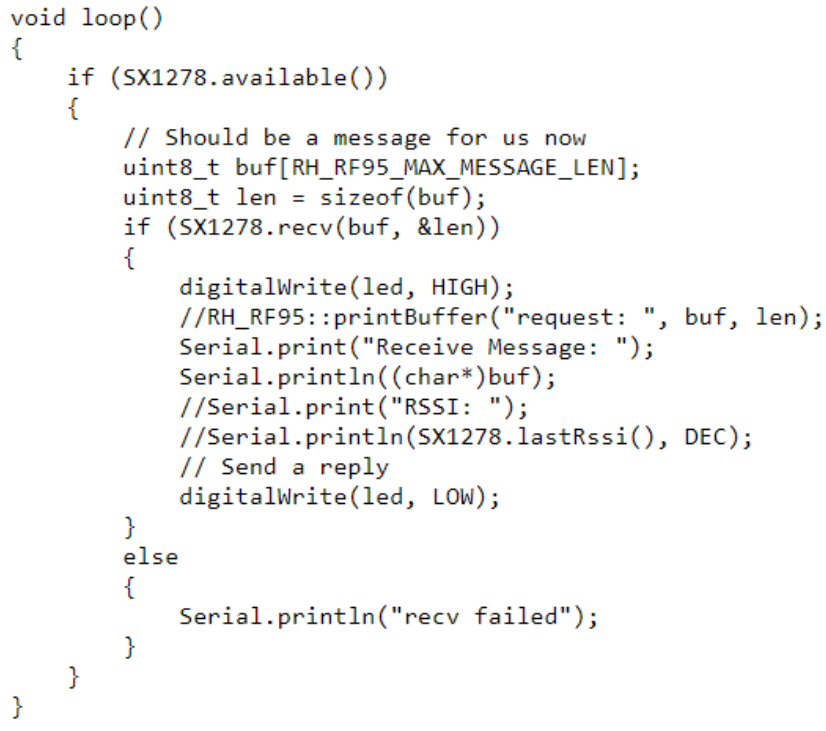

Figure 4. Part of the control software for the Receiver

Control algorithms (Fig. 5) were used to describe the control software. The left algorithm shows the sender's device side with the DHT11 sensor in first experiment, while the right side describes side of the receiver that was connected to a computer and provided the measured values.

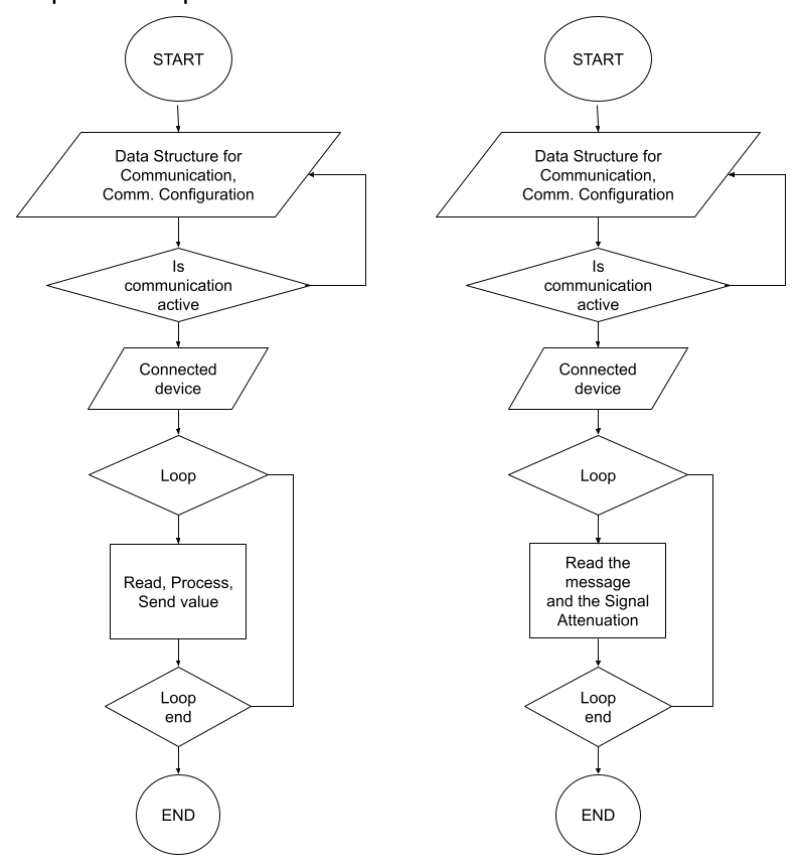

Figure 5. Control diagram

As already mentioned variable declarations and library calls are placed at the beginning of the code. These steps integrate the necessary functions to work with the connected input/output devices and communication modules. Variables define the memory space where potentiometer values, control values, communication variables are stored. The next step is to configure communication. The control loop follows which is repeated cyclically. 


\section{PROPOSED SYSTEM}

The tests were performed in laboratory environment on the premises of the Technical University of Kosice. Figure 6 below describes the actual connection of each pin on both sides of the LoRa system. As the picture shows the main control units are Arduino Rev3. During the experiments, the USB power supply cables were used on the sender's side and notebook USB port on the receiver's side. The USB connection allows the monitoring of various values such as humidity, temperature and fast software upload.

The final connection of the connected parts is shown on Fig. 6 . The first set is the sender's side, the measuring side, and the second set is the receiver with the notebook connected to monitor the received messages.

Long distance wireless connections have been established for testing. To evaluate the connection, we compared the data sent and received. Testing was successful. Due to the definition of communication protocols, while the transmitting parts are within range no communication error occurs. The experiments were focused on communication and finding out the reach of the two described protocol. The LoRa protocol offers Signal Attenuation measurement, the results of these measurements are in the following graphs (Fig.7). The ESPNow is currently in development and signal strength measurement is not yet supported.
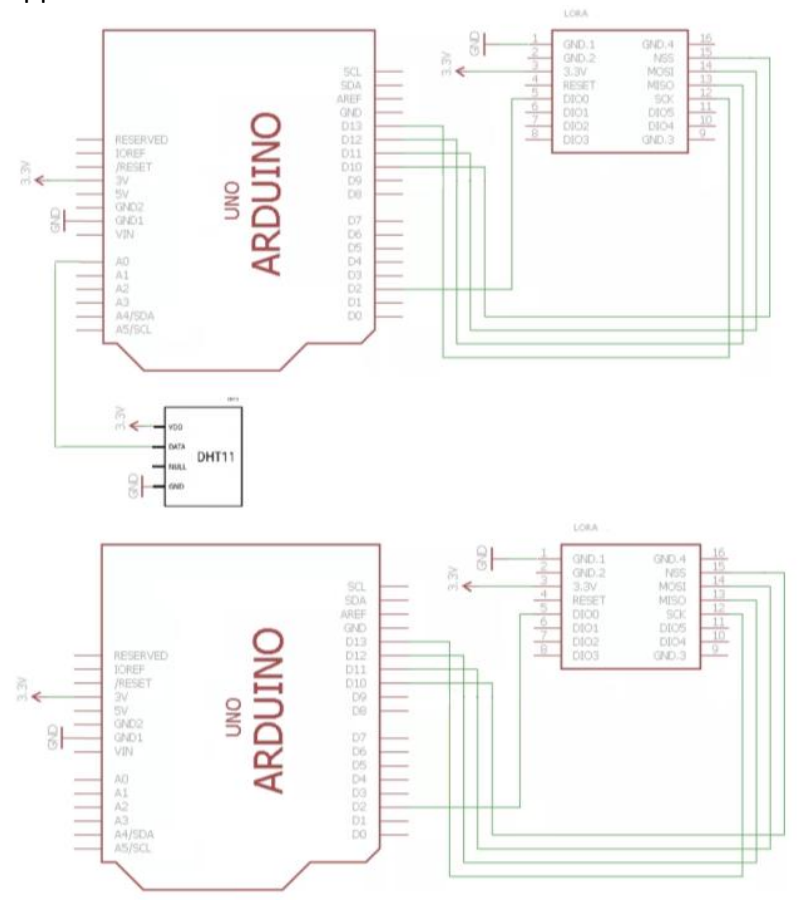

Figure 6. Wiring of the system

\section{MEASUEREMENT RESULTS}
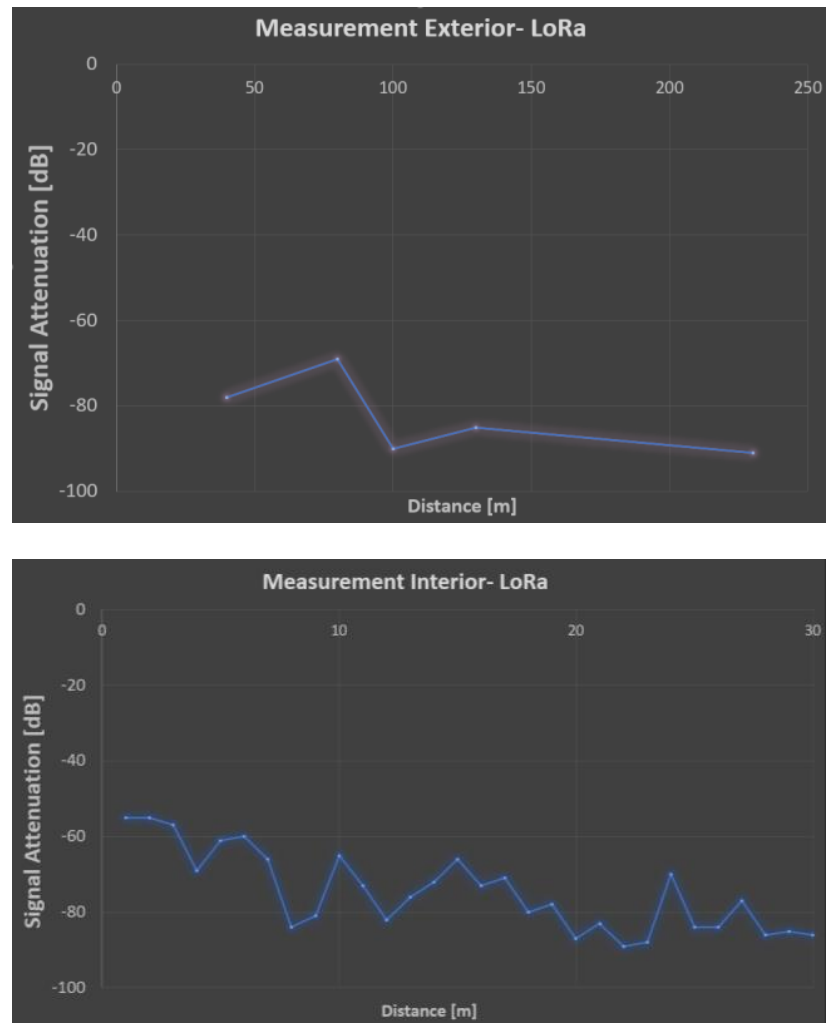

Figure 7. LoRa Measurement results

The second tested protocol, ESPNow, doesn't support RSSI measurement, so only the received message was a sign of a functioning communication.

However with this protocol we successfully transmitted data to a distance of more than $1.08 \mathrm{~km}$, while with the LoRa protocol only to a distance $230 \mathrm{~m}$.
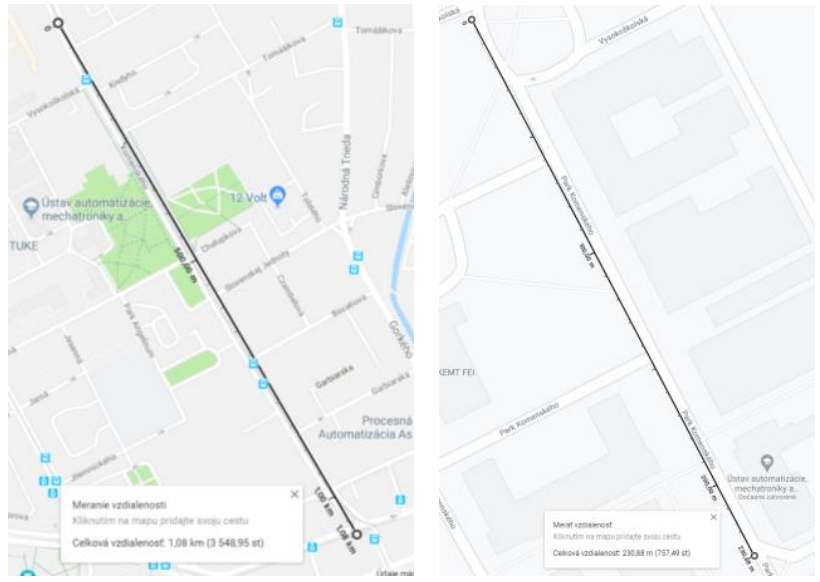

Figure 9. Wireless transmission reach ESPNow and LoRa

\section{CONCLUSIONS}

In this paper two wireless communication technologies were compared. The proposed systems of both protocols are suitable for home automation and/or monitoring systems for open spaces for asset monitoring, measurement of various parameters such as temperature, humidity, vibrations etc. With combination of batteries, both can be used as a good basis for 
such a monitoring system. To meet different requirements in real life, it is necessary to connect different input and output devices, based on the solved task, and analyze power consumption. In the tested systems we focused on testing the range of communication. The LoRa and the ESPNow communication protocols are similar transmission technologies suitable for IOT applications. Their characteristics are suitable for simple home automation systems, where one or more parameters are measured, and the communication is not time or content critical. In the first experiment a DHT11 sensor was used to generate message input and in the second experiment, a simple text messages were sent.

Experiments have shown that by connecting the LoRa module it is possible to create a smooth communication channel up to a distance $230 \mathrm{~m}$, while the ESPNow module can operate up to a distance $1 \mathrm{~km}$. However, ESP showed very high sensitivity to orientation which is a major disadvantage. But it can be used to connect two fixed objects at greater distances. Other big advantage of ESPNow is that it uses integrated antennas, and no additional hardware is required for communication. By combining batteries with these systems, we can create simple powerful micro automated systems. Compared to commercially available systems the proposed solution offers advantages such as reconfigurability with various peripherals, affordability, reuse in various tasks and provides good basis for acquiring knowledge in education of students in field of automation and mechatronics.

\section{ACKNOWLEDGMENTS}

This work has been supported by the Slovak Grant VEGA 1/0330/19 Research and design of algorithms and systems for fusion of disparate data in multisensor architectures (Vyskum a navrh algoritmov a systemov pre fúziu rôznorodych dat $v$ multisenzorovych architektúrach) and H2020: Manufacturing Industry Digital Innovation Hubs (MIDIH), reference No. 767498

\section{REFERENCES}

[Arduino 2021] Arduino UNO REV 3 Datasheet (2021), Available from: $\quad<$ https://store.arduino.cc/arduino-unorev3https://store.arduino.cc/arduino-uno->.

[ESP32 Datasheet 2021] ESP32 Datasheet (2021) Available from<http://

https://www.espressif.com/sites/default/files/documentation/ esp_wroom_32_datasheet_en.pdf>.

[ESPNow - Overview 2021] ESPNow - Overview (2021), Available from: <http://esp-idf.readthedocs.io/en/latest/apireference/wifi/esp_now.html\#>.

[Galajdova 2009] Galajdova, A., Simsik, D., Dolina, Z. Testing of E-services for Seniors and People with Disabilities within Einclusion Project MonAMI. International Journal of Rehabilitation Research, 2009, Vol. 32, pp. 75-76. ISSN 03425282.
[Kar 2017] Kar, U.N., Sanyan, D.K. An overview of device-todevice communication in cellular networks. ICT express. Available

from: <https://www.sciencedirect.com/science/article/pii/S24059595 17301467>.

[Ketshabetswe 2019] Ketshabetswe, L.K. et al. Communication protocols for wireless sensor networks: A survey and comparison. Heliyon, 2019 Available from: <https://www.sciencedirect.com/science/article/pii/S24058440 18340192?via\%3Dihub>.

[Krenicky 2011] Krenicky, T. Implementation of Virtual Instrumentation for Machinery Monitoring. In: Scientific Papers: Operation and Diagnostics of Machines and Production Systems Operational States: Vol. 4, RAM-Verlag, Lüdenscheid, 2011, pp. 5-8. ISBN 978-3-942303-10-1.

[LoRa-01 - Overview 2021] LoRa-01 - Overview (2021) Available from:

$<$ https://components101.com/asset/sites/default/files/compo nent_datasheet/SX1278\%20Lora\%20Datasheet.pdf>.

[Sarga 2018] Sarga, P., Cocula, J. Design and implementation of smart home model. Technical Sciences and Technologies, 2018, Vol. 13, No. 3, pp. 161-166. ISSN 2411-5363.

[Simsik 2016] Simsik, D., Galajdova, A., Seminsky, J., Rakay, R., Visňovsky, M. Innovation of the study program automation and control of machines and processes in response to the requirements of industrial practice (Inovacia studijneho programu automatizacia a riadenie strojov a procesov $\mathrm{v}$ reakcii na požiadavky priemyselnej praxe). ARaP, 2016, Prague: MM publishing, pp. 79-82. ISBN 978-80-906310-1-4. (in Slovak)

[Vagas 2020] Vagas, M., Galajdova, A., Simsik, D. Techniques for Secure Automated Operation with Cobots Participation. Institute of Electrical and Electronics Engineers, 2020, pp. 1-4. ISBN 978-1-7281-1951-9.

\section{CONTACTS}

Prof. MSc. Alena Galajdova, PhD.

Technical University of Kosice, Faculty of Mechanical Engineering,

Department of Automation and Human Machine Interactions, Park Komenskeho 8, 04200 Kosice, Slovakia alena.galajdova@tuke.sk

\section{MSc. Robert Rakay, PhD.}

Technical University of Kosice, Faculty of Mechanical Engineering,

Department of Automation and Human Machine Interactions, Park Komenskeho 8, 04200 Kosice, Slovakia

robert.rakay@tuke.sk 